\title{
Potencial antioxidante de extratos de sementes de limão (Citrus limon)
}

\author{
Antioxidant potential of lemon seed extracts (Citrus limon)
}

\author{
Débora Maria Moreno LUZIA ${ }^{1}$, Neuza JORGE ${ }^{1 *}$
}

\section{Resumo}

O presente trabalho teve como principais objetivos avaliar o comportamento do óleo de soja acrescido de extratos de sementes de limão (Citrus limon), em diferentes concentrações, por meio da estabilidade oxidativa e medir a atividade antioxidante através do método do radical livre DPPH e compostos fenólicos totais. A concentração de $2.400 \mathrm{mg} \cdot \mathrm{kg}^{-1}$ para o extrato de sementes de limão, variedade galego, foi a que conferiu melhor estabilidade oxidativa ao óleo de soja. As atividades antioxidantes máximas e os valores da concentração de extrato natural suficientes para obter 50\% do efeito máximo, estimado em 100\% $\left(\mathrm{EC}_{50}\right)$, determinados pelo DPPH para o extrato e ácido gálico foram 70,58\%, 69,94 $\mu \mathrm{g} \cdot \mathrm{mL}^{-1}$ e 75,07\%, 64,73 $\mu \mathrm{g} \cdot \mathrm{mL}^{-1}$, respectivamente. A concentração de compostos fenólicos totais, determinada pelo método de Folin-Ciocalteu foi de $76 \mathrm{mg} . \mathrm{g}^{-1}$. Foi possível concluir que o extrato de sementes de limão galego possui ação antioxidante natural, podendo ser aplicado em alimentos.

Palavras-chave: Citrus limon; antioxidante natural; compostos fenólicos totais; estabilidade oxidativa; sementes.

\begin{abstract}
The present study aimed at evaluating the behavior of the soybean oil with the addition of lemon seed extracts (Citrus limon), in different concentrations, through oxidative stability and also at measuring the antioxidant activity using the DPPH free radical method and total phenolic compounds. The concentration of $2,400 \mathrm{mg} \cdot \mathrm{kg}^{-1}$ for the lemon seed extract, galego variety, was the one that provided the soybean oil with the best oxidative stability. The maximum antioxidant activities and the concentration values of the natural extract sufficient to obtain $50 \%$ of maximum effect, estimated at $100 \%\left(\mathrm{EC}_{50}\right)$, determined by DPPH for the extract and gallic acid were $70.58 \%, 69.94 \mu \mathrm{g} . \mathrm{mL}^{-1}$ and $75.07 \%$, $64.73 \mu \mathrm{g} \cdot \mathrm{mL}^{-1}$, respectively. The concentration of total phenolic compounds, determined by the Folin-Ciocalteu method, was of 76 mg.g ${ }^{-1}$. Thus, it can be said that the lemon seed extract, galego, presents natural antioxidant action demonstrating potential to be used in foods. Keywords: Citrus limon; natural antioxidant; total phenolic compounds; oxidative stability; seeds.
\end{abstract}

\section{Introdução}

O retardo ou a prevenção da oxidação lipídica, uma das principais causas de deterioração no processo de aquecimento de óleos vegetais, pode ser realizado pela adição de antioxidantes, que mantêm a qualidade e prolongam a vida de prateleira do alimento (RAMALHO; JORGE, 2006).

No entanto, o emprego de antioxidantes sintéticos na indústria de alimentos tem sido alvo de questionamentos quanto à sua inocuidade. Sendo assim, pesquisas são realizadas para a busca de compostos naturais que apresentem esta propriedade funcional, podendo atuar como alternativa para prevenir a deterioração oxidativa de alimentos e diminuir o uso dos antioxidantes sintéticos. Os antioxidantes naturais podem ser encontrados e isolados em uma variedade de alimentos. Dentre as fontes de antioxidantes naturais podem ser citados os cereais, os cogumelos, as ervas e especiarias e as sementes de frutas cítricas (ELMASTAS, 2007; PEREIRA, 1996; PÉREZ-JIMÉNEZ; SAURA-CALIXTO, 2005; YANISHLIEVA; MARINOVA; POKORNÝ, 2006).

As substâncias presentes nessas fontes naturais, que são capazes de agir como antioxidantes, são minerais (principalmente constituindo enzimas), vitaminas e compostos fenólicos. Dentre os mais importantes, sob o ponto de vista tecnológico, podem ser citados os tocoferóis, os carotenoides, alguns ácidos orgânicos como o ácido cítrico e o ácido ascórbico, e os flavonoides. Os extratos de frutas cítricas, como o limão, são importantes fontes de ácidos fenólicos, como o ácido hidroxicinâmico, e de flavonoides (flavononas e flavonóis) (DIMITRIOS, 2006; ECONOMOS; CLAY, 1999). Esses compostos podem agir como redutores, interruptores de radicais livres, inibidores ou supressores de oxigênio singlete e como inativadores de metais pró-oxidantes.

Existem diversos métodos utilizados para a identificação e quantificação desses antioxidantes naturais. Dentre eles podem ser citados o método TBA (valor do ácido tiobarbitúrico), a determinação dos compostos fenólicos totais, o sistema do $\beta$-caroteno/ácido linoleico, a Cromatografia Líquida de Alta Eficiência (CLAE) e os métodos de detecção de sequestradores de radicais livres, como o 2,2-azino-bis (3-etilbenzotiazolina-6-sulfônico) (ABTS) e o 2,2-difenil-1-picrilhidrazil (DPPH).

Recebido para publicação em 11/7/2008

Aceito para publicação em 16/5/2009 (003669)

${ }^{1}$ Departamento de Engenharia e Tecnologia de Alimentos, Instituto de Biociências, Letras e Ciências Exatas, Universidade do Estado de São Paulo - UNESP,

Rua Cristóvão Colombo, 2265, Jd. Nazareth, CEP 15054-000, São José do Rio Preto - SP, Brasil, E-mail: njorge@ibilce.unesp.br

${ }^{*}$ A quem a correspondência deve ser enviada 
No teste do DPPH, a ação do radical DPPH é acompanhada pelo monitoramento da diminuição da absorbância a $515 \mathrm{~nm}$, que ocorre devido a sua reação com algum antioxidante ou com algum radical livre (BRAND-WILLIAMS et al., 1995).

O DPPH é um radical livre, estável à temperatura ambiente, que produz uma coloração violeta quando em contato com etanol. Este radical é reduzido na presença de uma molécula de antioxidante doador de hidrogênio. O DPPH captura os hidrogênios mudando a coloração de violeta para amarelo, passando para sua forma estável DPPH-H. O radical DPPH mostra forte banda de absorção em $515 \mathrm{~nm}$.

Alguns estudos demonstraram que a interação entre antioxidante e DPPH depende de sua conformação estrutural. $\mathrm{O}$ número de moléculas de DPPH reduzidas está relacionado com o número de grupos hidroxilas disponíveis no composto antioxidante (BRAND-WILLIAMS et al., 1995).

Os compostos fenólicos funcionam como sequestradores de radicais livres e, algumas vezes, como quelantes de metais (SHAIDI; JANITHA; WANASUNDARA, 1992), agindo tanto na etapa de iniciação como na propagação do processo oxidativo.

Assim, os principais objetivos deste trabalho foram avaliar o comportamento do óleo de soja acrescido de extratos de sementes de limão, em diferentes concentrações, por meio da estabilidade oxidativa e medir a atividade antioxidante através do método do radical livre DPPH e compostos fenólicos totais presentes no extrato.

\section{Material e métodos}

\subsection{Material}

Óleo

Para a realização deste trabalho, utilizou-se óleo de soja refinado, sem adição de antioxidantes sintéticos (TBHQ e ácido cítrico), adquirido em embalagens de $900 \mathrm{~mL}$, processado pela empresa Cargill Agrícola S/A, Uberlândia-MG.

\section{Sementes de limão}

As frutas maduras, variedades cravo e galego, foram provenientes de uma plantação na região de Itajobi-SP, colhidas em Janeiro de 2007. Os limões foram cortados pela metade e as sementes retiradas manualmente e, em seguida, lavadas ligeiramente com água destilada para remover restos de polpas e açúcares solúveis provenientes das frutas. As sementes foram secas em estufa, com circulação de ar forçada, a $45{ }^{\circ} \mathrm{C}$ por 24 horas para redução do teor de umidade abaixo de $10 \%$. Depois, foram armazenadas em recipientes plásticos, vedados com tampas de rosca e devidamente rotulados, para análises posteriores.

\section{Extratos de sementes de limão}

Os extratos metanólicos de sementes de limão, cravo e galego, foram obtidos de acordo com a metodologia descrita por Pereira (1996). As sementes de limão desidratadas e trituradas $(10 \mathrm{~g})$ foram mantidas sob agitação permanente com metanol
$(100 \mathrm{~mL})$ à temperatura ambiente $\left(25 \pm 2{ }^{\circ} \mathrm{C}\right)$ durante 6 horas e, em seguida, a mistura foi centrifugada a $3.000 \mathrm{rpm}$, por 10 minutos, e o sobrenadante filtrado em filtro comum. Após a transferência do sobrenadante, o precipitado foi novamente submetido ao processo de extração nas mesmas condições anteriormente explicitadas, e os sobrenadantes resultantes das duas extrações foram combinados. Em seguida, procedeu-se à remoção dos solventes utilizados para a obtenção dos extratos metanólicos, sob pressão reduzida a $40{ }^{\circ} \mathrm{C}$. Os extratos secos foram pesados e ressuspensos em metanol, obtendo-se soluções-estoques contendo um grama de extrato metanólico para cada dez g de solvente metanol (1:10), utilizadas para aplicações diretas no óleo de soja.

\subsection{Métodos}

\section{Estabilidade oxidativa}

Os extratos de sementes de limão foram aplicados ao óleo de soja em diferentes concentrações $(0,500,1000,1500,2000$ e $2500 \mathrm{mg} \cdot \mathrm{kg}^{-1}$ ) com o objetivo de avaliá-los quanto à estabilidade oxidativa. As amostras foram analisadas segundo o método proposto pela American Oil Chemist's Society Cd 12-92 (1993), utilizando o equipamento Rancimat ${ }^{\oplus}$ modelo 743 , marca Metrohm, nas seguintes condições: $3,0 \mathrm{~g}$ de óleo; fluxo de ar

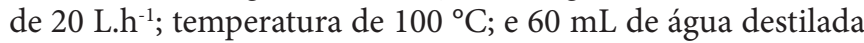
nos frascos contendo os eletrodos. Essa medida se baseia na determinação da condutividade elétrica dos produtos voláteis de degradação.

A amostra, cuja variedade e concentração do extrato foi considerada de maior eficiência contra a oxidação lipídica, foi aquela que apresentou maior período de indução, em horas. Em seguida, foi avaliada quanto à sua atividade antioxidante pelo método de DPPH e compostos fenólicos totais.

\section{Método do radical livre DPPH}

$\mathrm{O}$ teor de antioxidantes foi determinado, segundo a metodologa descrita por Brand-Williams, Cuvelier e Berset (1995). Neste procedimento, preparou-se uma solução metanólica de $500 \mu \mathrm{g} \cdot \mathrm{mL}^{-1}$ de concentração de extrato de sementes de limão (solução mãe), a partir da qual se obtiveram soluções de $5,10,25,50,125$ e $250 \mu \mathrm{g} \cdot \mathrm{mL}^{-1}$. Alíquotas de cada uma destas soluções $(0,3 \mathrm{~mL})$ foram acrescidas de $2,7 \mathrm{~mL}$ de solução de DPPH $\left(40 \mu \mathrm{g} \cdot \mathrm{mL}^{-1}\right)$. Após o tempo de reação de 30 minutos, a absorbância foi lida em $515 \mathrm{~nm}$ e convertida em porcentagem de atividade antioxidante (AA) por meio da seguinte Equação 1:

AA $(\%)=100-\left\{\frac{\left[\left(\mathrm{Abs}_{\left.\left.\text {amostra }-\mathrm{Abs}_{\text {branco }}\right) \times 100\right]}\right.\right.}{\mathrm{Abs}_{\text {controle }}}\right\}$

onde Abs = absorbância.

Um controle foi feito com $2,7 \mathrm{~mL}$ de DPPH e o branco foi realizado com $0,3 \mathrm{~mL}$ de solução metanólica do extrato e $2,7 \mathrm{~mL}$ de metanol, para cada concentração.

Esta metodologia permite a determinação do valor $\mathrm{EC}_{50}\left(\mathrm{mg} \cdot \mathrm{L}^{-1}\right)$, definido como a concentração de extrato natural 
suficiente para atingir $50 \%$ da atividade antioxidante máxima, estimada em $100 \%$, obtida por regressão linear.

\section{Compostos fenólicos totais}

A quantificação de compostos fenólicos totais foi determinada por espectrofotometria, por meio do reagente de Folin-Ciocauteau, segundo a metodologia descrita por Singleton e Rossi (1965).

Neste procedimento, pipetou-se $100 \mu \mathrm{L}$ da solução de extrato natural em tubos de ensaio e adicionaram-se $500 \mu \mathrm{L}$ do reagente de Folin-Ciocalteau. Em seguida, adicionaram-se 1,5 mL de solução saturada de carbonato de sódio $20 \%$ e $6 \mathrm{~mL}$ de água destilada.

Essa mistura permaneceu em repouso por 2 horas em temperatura ambiente, e a absorbância foi determinada a 765 nm. Para a quantificação, foi feita uma curva de calibração, utilizando ácido gálico em concentrações de 0 a $500 \mathrm{mg} \cdot \mathrm{L}^{-1}$. O coeficiente de determinação da curva analítica foi de $\mathrm{R}^{2}=0,9986$.

\subsection{Análise estatística}

O experimento foi realizado em esquema fatorial $2 \times 6$, no delineamento inteiramente casualizado, em duas repetições (BANZATTO; KRONKA, 2006). Os resultados obtidos de duas determinações foram submetidos à análise de variância para estudar a interação entre as variedades e concentrações e a regressão polinomial, a fim de determinar a influência das concentrações sobre a estabilidade oxidativa do óleo de soja acrescido de extrato de sementes de limão, medida pelo Rancimat, em horas. A análise de variância e o teste de Tukey para as médias a $5 \%$ foram obtidos por meio do programa ESTAT - Sistema para Análises Estatísticas, versão 2.0 (UNIVERSIDADE ESTADUAL PAULISTA, 1999).

\section{Resultados e discussão}

\subsection{Rendimento dos extratos de sementes de limão}

As sementes frescas de limão in natura, variedades cravo e galego, apresentaram teores de umidade de 46,10 e 46,95\%, respectivamente, sendo estes valores próximos aos encontrados por Reda et al. (2005) para as sementes de limão rosa (48,00\%) e limão siciliano (48,30\%). Após a desidratação, para a obtenção dos extratos, os teores de umidade das sementes passaram a ser 7,57 e 6,62\%, para as sementes de limão cravo e galego, respectivamente.

A Tabela 1 apresenta o rendimento porcentual dos extratos obtidos após a remoção do solvente orgânico empregado no processo de extração. Observa-se que os rendimentos diferiram significativamente $(\mathrm{p}<0,05)$, e o maior rendimento em extrato seco foi obtido para a variedade de limão galego (12,47\%).

Os rendimentos, em porcentagem, dos extratos de sementes de limão foram próximos aos encontrados por Rehman (2006) que, em diferentes solventes orgânicos, obteve valores que variaram de 7,88 a $19,87 \%$ para os extratos de cascas de frutas cítricas.

\subsection{Estabilidade oxidativa}

Para determinar a concentração mais efetiva dos extratos testados, bem como uma possível ação pró-oxidante, foram aplicadas concentrações de 0, 500, 1000, 1500, 2000 e $2500 \mathrm{mg} \cdot \mathrm{kg}^{-1}$ dos extratos de sementes de limão, cravo e galego, no óleo de soja e a estabilidade oxidativa foi medida utilizando-se o Rancimat a $100{ }^{\circ} \mathrm{C}$.

De acordo com a Tabela 2, observa-se que, dentre as variedades estudadas, o limão galego apresentou maior estabilidade oxidativa quando comparado com o limão cravo, sendo a diferença evidenciada estatisticamente, conforme teste de Tukey $(\mathrm{p}<0,05)$.

Verifica-se que houve efeito protetor contra a oxidação lipídica quando foram empregadas concentrações de 500 a 2500 mg. $\mathrm{kg}^{-1}$. A estabilidade oxidativa foi diretamente proporcional ao aumento da concentração do extrato de sementes de limão. Porém, as concentrações a partir de $1500 \mathrm{mg} \cdot \mathrm{kg}^{-1}$ apresentaram maior efeito protetor ao óleo de soja, apesar de não diferirem significativamente pelo teste de Tukey $(\mathrm{p}>0,05)$.

Em geral, as mesmas características são encontradas na literatura para a ação antioxidante de extratos naturais, ou seja, a tendência é o aumento da atividade antioxidante, conforme o aumento da concentração de extrato (AZIZAH; RUSLAWATI; TEE, 1999; LEE; KIM; ASHMORE, 1986).

Por meio da regressão polinomial (Figura 1), observa-se que a maior atividade antioxidante, medida pela estabilidade oxidativa, foi para a concentração de $2.400 \mathrm{mg} \cdot \mathrm{kg}^{-1}$, obedecendo, assim, a equação de $2^{\circ}$ grau (Equação 2).

$y=-1 E-06 x^{2}+0,0048 x+11,075$

Tabela 1. Rendimento dos extratos de sementes de limão, variedades cravo e galego, obtidos por extração com metanol.

\begin{tabular}{ccc}
\hline Solvente orgânico & \multicolumn{2}{c}{ Rendimento (\%)* } \\
\cline { 2 - 3 } & Cravo & Galego \\
\hline Metanol & $11,18^{\mathrm{b}} \pm 0,14$ & $12,47^{\mathrm{a}} \pm 0,14$ \\
\hline
\end{tabular}

*Valores médios \pm desvio padrão seguidos por diferentes letras diferem pelo teste de Tukey $(\mathrm{p}<0,05)$.

Tabela 2. Médias da estabilidade oxidativa para os fatores Variedades e Concentrações.

\begin{tabular}{|c|c|}
\hline Fatores & Estabilidade Oxidativa (horas)* \\
\hline \multicolumn{2}{|l|}{ Variedades } \\
\hline Limão Cravo & $13,74^{\mathrm{b}}$ \\
\hline Limão Galego & $14,37^{\mathrm{a}}$ \\
\hline \multicolumn{2}{|l|}{ Concentrações $\left(\mathrm{mg} \cdot \mathrm{kg}^{-1}\right)^{\star \star}$} \\
\hline 0 & $10,52^{c}$ \\
\hline 500 & $13,84^{\mathrm{b}}$ \\
\hline 1000 & $14,32^{\mathrm{b}}$ \\
\hline 1500 & $14,98^{\mathrm{a}}$ \\
\hline 2000 & $15,21^{\mathrm{a}}$ \\
\hline 2500 & $15,47^{\mathrm{a}}$ \\
\hline
\end{tabular}

\footnotetext{
${ }^{*}$ Valores médios seguidos pelas mesmas letras não diferem pelo teste de Tukey $(\mathrm{p}>0,05)$.
}

**mg extrato de sementes de limão por kg de óleo de soja. 
onde y é a estabilidade oxidativa (horas) e $\mathrm{x}$ as concentrações dos extratos de sementes de limão $\left(\mathrm{mg} \cdot \mathrm{kg}^{-1}\right)$. De acordo com a análise de variância, para os extratos, a regressão cúbica foi significativa $(\mathrm{p}<0,01)$, porém o coeficiente de deteminação da regressão quadrática $\left(\mathrm{R}^{2}=0,9282\right)$ foi maior que 0,9 , sendo, portanto, utilizada para explicar o comportamento dos extratos de sementes de limão.

Com base na estabilidade oxidativa, a amostra correspondente à variedade limão galego, na concentração de $2.400 \mathrm{mg} \cdot \mathrm{kg}^{-1}$, foi a que teve maior atividade antioxidante (16,84 horas), passando, então, a ser avaliada quanto à sua atividade antioxidante pelo método de DPPH e compostos fenólicos totais.

\subsection{Atividade antioxidante}

A atividade antioxidante do extrato de sementes de limão galego foi determinada pelo método do radical DPPH. Esta metodologia permite a avaliação do comportamento antioxidante dos compostos através da capacidade de sequestrar radical livre em determinado período de tempo. A atividade antioxidante dos compostos, representada pelo valor de $\mathrm{EC}_{50}$, é calculada pela redução de $50 \%$ da concentração inicial de $\mathrm{DPPH}$. Ressalta-se que, quanto menor o valor de $\mathrm{EC}_{50}$, maior a atividade antioxidante do composto analisado.

A Tabela 3 ilustra a porcentagem máxima de atividade antioxidante $\mathrm{AA}_{\text {máxima }}(\%)$ e o valor de $\mathrm{EC}_{50}\left(\mu \mathrm{g} \cdot \mathrm{mL}^{-1}\right)$ Os valores de $\mathrm{EC}_{50}$, obtidos por regressão linear, para o extrato de sementes de limão galego e ácido gálico, apresentavam bons coeficientes de determinação, 0,9109 e 0,9115, respectivamente. As atividades antioxidantes máximas atingidas para extrato e ácido gálico foram 70,58 e 75,07\%, e para $\mathrm{EC}_{50}$ foram 69,94 e $64,73 \mu \mathrm{g} \cdot \mathrm{mL}^{-1}$, respectivamente. Observa-se, que o extrato de

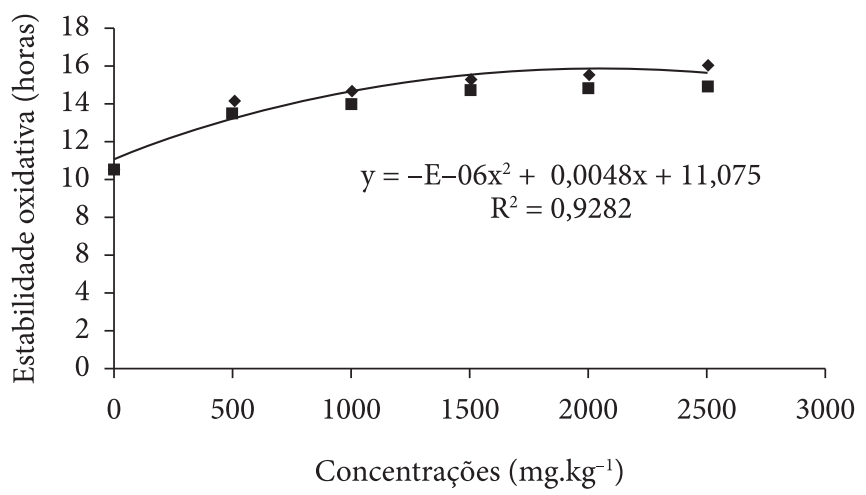

Figura 1. Regressão polinomial para os extratos de sementes de limão.

Tabela 3. Valor, em porcentagem, de $\mathrm{AA}_{\text {máxima }}$ e $\mathrm{EC}_{50}$ para o extrato de sementes de limão galego (ESLG) e ácido gálico (AG).

\begin{tabular}{ccc}
\hline Antioxidante & $\mathrm{AA}_{\text {máxima }}(\%)^{*}$ & $\mathrm{EC}_{50}\left(\mu \mathrm{g} \cdot \mathrm{mL}^{-1}\right)^{\star}$ \\
\hline ESLG & $70,58^{\mathrm{b}} \pm 0,14$ & $69,94^{\mathrm{a}} \pm 0,14$ \\
$\mathrm{AG}$ & $75,07^{\mathrm{a}} \pm 0,14$ & $64,73^{\mathrm{b}} \pm 0,14$ \\
\hline
\end{tabular}

*Valores médios \pm desvio padrão seguidos por diferentes letras nas colunas diferem pelo teste de Tukey ( $\mathrm{p}<0,05) ; \mathrm{EC}_{50}$ é definido como a concentração suficiente para obter 50\% do efeito máximo, estimado em $100 \%$. sementes de limão galego e ácido gálico, usado para comparação, diferiram significativamente pelo teste de Tukey $(\mathrm{p}<0,05)$.

Em estudo realizado por Sousa et al. (2007), foi demonstrado que os extratos de plantas medicinais e o ácido gálico, usado para comparação, apresentaram capacidade em sequestrar o radical DPPH, obtendo-se, assim, atividades antioxidantes que variaram de 10 a $91 \%$ para os extratos de plantas medicinais, e o ácido gálico obteve $94,84 \%$.

A concentração de compostos fenólicos totais encontrada foi de $76 \mathrm{mg}$ de equivalentes de ácido gálico por grama de extrato.

Os resultados obtidos neste trabalho foram similares aos encontrados na literatura. Wang, Chuang e Ku (2007) encontraram teor de compostos fenólicos para limão de 75,9 mg de equivalentes de ácido gálico por grama de extrato, enquanto para Li, Smith e Hossain (2006), os resultados para teores de compostos fenólicos extraídos de cascas de citrus por 6 horas, apresentaram 70,43 mg de equivalentes de ácido gálico por grama de extrato.

A extração de compostos fenólicos de produtos naturais é fortemente influenciada pelo solvente utilizado na extração. Tem-se observado que, quanto maior a polaridade do solvente de extração, maior a quantidade de compostos fenólicos extraídos (GAMÉZ-MEZA et al., 1999).

\section{Conclusões}

Entre as concentrações avaliadas neste estudo, $2.400 \mathrm{mg} \cdot \mathrm{kg}^{-1}$ de extrato de sementes de limão galego promoveram maior estabilidade oxidativa ao óleo de soja, medida por meio do Rancimat. $\mathrm{O}$ extrato de sementes de limão galego, devido à sua atividade antioxidante, apresenta-se como uma alternativa natural para ser aplicado em alimentos industrializados como antioxidante natural.

\section{Agradecimentos}

À Coordenação de Aperfeiçoamento de Pessoal de Nível Superior - CAPES, pela concessão da bolsa de Mestrado, e ao Conselho Nacional de Desenvolvimento Científico e Tecnológico - CNPq, pela bolsa de Produtividade em Pesquisa.

\section{Referências bibliográficas}

AMERICAN OIL CHEMISTS' SOCIETY. Official methods and recommended practices of the American Oil Chemists' Society. Champaign: AOCS, 1993.

AZIZAH, A. H.; RUSLAWATI, N. M. N.; TEE, T. S. Extraction and characterization of antioxidant from cocoa by-products. Food Chemistry, v. 64, n. 2, p. 199-202, 1999.

BANZATTO, D. A.; KRONKA, S. N. Experimentação agrícola. 4. ed. Jaboticabal: FUNEP, 2006. 237 p.

BRAND-WILLIAMS, W.; CUVELIER, M. E.; BERSET, C. Use of a free radical method to evaluate antioxidant activity. Lebensmittel Wissenschaft Technologie, v. 28, n. 1, p. 25-30, 1995.

DIMITRIOS, B. Sources of natural phenolics antioxidants. Trends in Food Science \& Technology, v. 17, n. 9, p. 505-512, 2006. 
ECONOMOS, C.; CLAY, W. D. Nutritional and health benefits of citrus fruits. Food, Nutrition and Agriculture, v. 24, n. 1, p. 8-11, 1999.

ELMASTAS, M. et al. Determination of antioxidant activity and antioxidant compounds in wild edible mushrooms. Journal of Food Composition and Analysis, v. 20, n. 3, p. 337-345, 2007.

GÁMEZ-MEZA, N. et al. Antioxidant activity in soybean oil of extracts from Thompson grape bagasse. Journal of the American Oil Chemist's Society, v. 76, n. 12, p. 1445-1447, 1999.

LEE, Y. B.; KIM, Y. S.; ASHMORE, C. R. Antioxidant properties in ginger rhizome and its application to meat products. Journal of Food Science, v. 51, n. 1, p. 20-23, 1986.

LI, B. B.; SMITH, B.; HOSSAIN, M. M. Extraction of phenolics from citrus peels: solvent extraction method. Separation and Purification Technology, v. 48, n. 2, p. 182-188, 2006.

PEREIRA, R. B. Avaliação da atividade antioxidante de sementes de frutas cítricas. 1996. 90 f. Dissertação (Mestrado) - Faculdade de Ciências Farmacêuticas, Universidade de São Paulo, São Paulo, 1996.

PÉREZ-JIMÉNEZ, J.; SAURA-CALIXTO, F. Literature data may underestimate the actual activity of cereals. Journal of Agricultural and Food Chemistry, v. 53, n. 13, p. 5036-5040, 2005.

RAMALHO, V. C.; JORGE, N. Antioxidantes utilizados em óleos, gorduras e alimentos gordurosos. Química Nova, v. 29, n. 4, p. 755-760, 2006.
REDA, S. Y. et al. Caracterização dos óleos das sementes de limão rosa (Citrus limonia Osbeck) e limão siciliano (Citrus limon), um resíduo agroindustrial. Ciência e Tecnologia de Alimentos, v. 25, n. 4, p. 672-676, 2005.

REHMAN, Z. Citrus peel extract: a natural source of antioxidant. Food Chemistry, v. 99, n. 3, p. 450-454, 2006.

SHAIDI; F.; JANITHA, P. K.; WANASUNDARA, P. D. Phenolic antioxidants. Critical Reviews in Food Science and Nutrition, v. 32, n. 1, p. 67-103, 1992.

SINGLETON, V. L.; ROSSI, J. A. Colorimetry of total phenolics with phosphomolybdic-phosphotungstic acid reagents. American Journal of Enology and Viticulture, v. 16, n. 3, p. 144-158, 1965.

SOUSA, C. M. M. et al. Fenóis totais e atividade antioxidante de cinco plantas medicinais. Química Nova, v. 30, n. 2, p. 351-355, 2007.

UNIVERSIDADE ESTADUAL PAULISTA - UNESP. Faculdade de Ciências Agrárias e Veterinárias. ESTAT: sistema para análises estatísticas. Jaboticabal, 1999. Versão 2.0. 1 disquete.

WANG, Y. C.; CHUANG, Y. C; KU, Y. H. Quantitation of bioactive compounds in citrus fruits cultivated in Taiwan. Food Chemistry, v. 102, n. 4, p. 1163-1171, 2007.

YANISHLIEVA, N. V.; MARINOVA, E.; POKORNÝ, J. Natural antioxidants from herbs and spices. European Journal of Lipids Science and Technology, v. 108, n. 9, p. 776-793, 2006. 